\title{
Macrophage-derived extracellular vesicles: diverse mediators of pathology and therapeutics in multiple diseases
}

Yizhuo Wang ${ }^{1}$, Meng Zhao', Shuyun Liu ${ }^{1,2}$, Jun Guo ${ }^{3}$, Yanrong Lu ${ }^{1,2}$, Jingqiu Cheng ${ }^{1,2}$ and Jingping Liu ${ }^{1,2}$

\begin{abstract}
Macrophages $(M \varphi)$ are primary innate immune cells that exhibit diverse functions in response to different pathogens or stimuli, and they are extensively involved in the pathology of various diseases. Extracellular vesicles (EVs) are small vesicles released by live cells. As vital messengers, macrophage-derived EVs ( $M \varphi$-EVs) can transfer multiple types of bioactive molecules from macrophages to recipient cells, modulating the biological function of recipient cells. In recent years, M $\varphi$-EVs have emerged as vital mediators not only in the pathology of multiple diseases such as inflammatory diseases, fibrosis and cancers, but also as mediators of beneficial effects in immunoregulation, cancer therapy, infectious defense, and tissue repair. Although many investigations have been performed to explore the diverse functions of $M \varphi$-EVs in disease pathology and intervention, few studies have comprehensively summarized their detailed biological roles as currently understood. In this review, we briefly introduced an overview of macrophage and EV biology, and primarily focusing on current findings and future perspectives with respect to the pathological and therapeutic effects of $M \varphi$-EVs in various diseases.
\end{abstract}

\section{Facts}

- EVs can carry and transfer various bioactive molecules towards recipient cells and thus participate in the cell-cell communication during disease pathology and tissue regeneration.

- Macrophages play essential roles in the pathology of multiple diseases, and their released EVs are also believed to participate in these courses.

- M $\phi$-EVs can serve as potential therapeutic targets as
Correspondence: Yanrong Lu (luyanrong@scu.edu.cn) or Jingqiu Cheng (jqcheng@scu.edu.cn) or Jingping Liu (liujingping@scu.edu.cn) ${ }^{1}$ Key Laboratory of Transplant Engineering and Immunology, Regenerative Medicine Research Center, National Clinical Research Center for Geriatrics, West China Hospital, Sichuan University, Chengdu, China

${ }^{2}$ Institutes for Systems Genetics, Frontiers Science Center for Disease-related Molecular Network, West China Hospital, Sichuan University, Chengdu, China Full list of author information is available at the end of the article These authors contributed equally: Yizhuo Wang, Meng Zhao Edited by T. Kaufmann well as promising agents for the treatment of diseases due to their similar abilities to macrophages.

\section{Open questions}

- How can we properly regulate the release or the embedded contents of $M \phi-E V s$ to prevent the development of diseases?

- How can we precisely modulate M $\phi$-EVs to exert diverse functions in response to different microenvironments or disease states?

- Is there any method to generate the reprogrammed/ reengineered $\mathrm{M} \phi-E V s$ with improved yield and biofunction?

\section{Introduction}

Macrophages are key innate immune cells that circulate in the blood and reside in nearly all tissues with selfrenewal capacity and tissue-specific characteristics ${ }^{1}$. Macrophages initially originate from highly heterogeneity 
hemopoietic progenitors to perform multiple functions that depend on the various stimuli. Macrophages constitute the first barrier against invading pathogens, which are activated and exhibit differential phenotypes in response to a variety of endogenous and exogenous danger signals, mediating the homeostasis of the immune system and multiple tissues. However, abnormal macrophage responses may induce immune disorders and uncontrolled inflammation, which have been implied in many diseases. For example, damage-associated molecular patterns (DAMPs) released after kidney injury activate toll-like receptors (TLRs) and nuclear factor- $\kappa B$ (NF- $\mathrm{KB}$ ) in macrophages, stimulating reactive oxygen species (ROS) and inflammatory cytokines release that further aggravates inflammation and renal injury ${ }^{2}$. Generally, activated macrophages communicate with various types of target cells to exert their immunomodulatory effects via direct cell-to-cell contact and/or release of the secretome including cytokines and extracellular vesicles (EVs).

EVs are a group of membrane-enclosed vesicles that are naturally released by nearly all types of cells. EVs can be divided into multiple subtypes, such as exosomes, microvesicles, apoptotic bodies, exomeres, and large oncosomes, based on their different origins and sizes. EVs package proteins, nucleic acids, and metabolites of parental cells, and are thought to exhibit similar properties to their parent cells. Recently, EVs have been recognized as vital information carriers that transfer their cargos from parent cells to recipient cells, modulating the physiological or pathological processes in recipient cells. The functions of EVs derived from macrophages in various disease states have been widely investigated, and increasing evidence indicates that these EVs play key roles in the diseases progression. Thus, a comprehensive understanding is needed of macrophage-derived EVs $(\mathrm{M} \phi$-EVs) and their roles in the disease pathology and treatment. In this review, we discussed the important studies with respect to the biological and therapeutic effects of EVs from macrophages in various diseases.

\section{Macrophage polarization and functions}

Macrophages are highly heterogeneous immune cells that act in response to various stimuli. Depending on the microenvironments, macrophages exhibit different phenotypes and can be roughly divided into two subtypes: classically activated macrophages (CAMs, M1-like macrophages) and alternatively activated macrophages (AAMs, M2-like macrophages) ${ }^{3-5}$. With the increasing understanding of macrophages, M2-like macrophages have been further subdivided into the M2a, M2b, M2c, and M2d subtypes based on their gene expression profiles. In general, M1-like macrophages comprise the majority population during early inflammation against danger signals, and then they skew towards M2-like macrophages exhibiting immunoregulatory effects to facilitate tissue repair, regeneration, and fibrosis ${ }^{6}$. The phenotype switch between M1-like and M2-like cells is intimately associated with the disease development. Although the functions of these two types of macrophages are largely distinct, they work collectively to regulate tissue homeostasis.

\section{M1-like macrophages}

M1-like macrophages play important roles during the early stage of pathogen invasion and inflammatory diseases and are typically induced by interferon- $\gamma$ (IFN- $\gamma$ ), TNF- $\alpha$, granulocyte monocyte colony-stimulating factor (GM-CSF), or lipopolysaccharide (LPS) in vitro. M1-like macrophages are characterized by several markers, such as CD86, CD68, TNF- $\alpha$, MHC class II molecules, inducible nitric oxide synthase (iNOS), NOS2, and suppressor of cytokine signaling 3 (SOCS3; Fig. 1). M1-like macrophages secrete high levels of proinflammatory cytokines such as interleukin-1 $\beta$ (IL-1 $\beta$ ), IL-6, IL-12, IL-23, and TNF- $\alpha$, promoting the inflammatory and cytotoxic responses ${ }^{7}$. In addition, M1-like macrophages generate high levels of reactive oxygen species (ROS) and reactive nitrogen species (RNS) to fight against invading pathogens $^{8}$. iNOS acts on L-arginine to produce nitric oxide (NO), which mediates antibacterial and antifungal responses 9 .

When foreign pathogens such as fungi, bacteria and parasites, M1 macrophages release proinflammatory cytokines and induce T helper 1 (Th1) cells differentiation, exhibiting enormous proinflammatory response to deal with these pathogens, which is beneficial for individuals fighting against early stage pathogens invasion. However, excessive proinflammatory and cytotoxic responses can cause severe tissue damage ${ }^{10}$. M1-like macrophages are involved in many inflammatory diseases such as rheumatoid arthritis, hepatitis, inflammatory bowel disease, metabolic syndrome, and diabetes.

\section{M2-like macrophages}

Compared to M1-like macropahges, M2-like macrophages are more likely to contribute trophism, phagocytosis, and induction of tissue tolerance rather than acting as efficient killers. M2-like macrophages are typically induced by $\mathrm{T}$ helper 2 (Th2) cytokines (Fig. 1). M2a macrophages are induced by IL- 4 and IL- 13 via activation of the STAT6 pathway through the common receptor IL$4 \mathrm{R} \alpha$, which primarily mediates tissue repair and antifungal response $^{11,12}$. Activation of STAT6 can induces the production of arginase-1 (Arg1), which then degrades arginine into polyamines and prolines, thereby promoting cell proliferation and collagen deposition for tissue repair. $\mathrm{M} 2 \mathrm{~b}$ macrophages are generated upon exposure to immune complexes and IL-1R or TLR agonists and primarily participate in regulating the immune response ${ }^{13}$. 


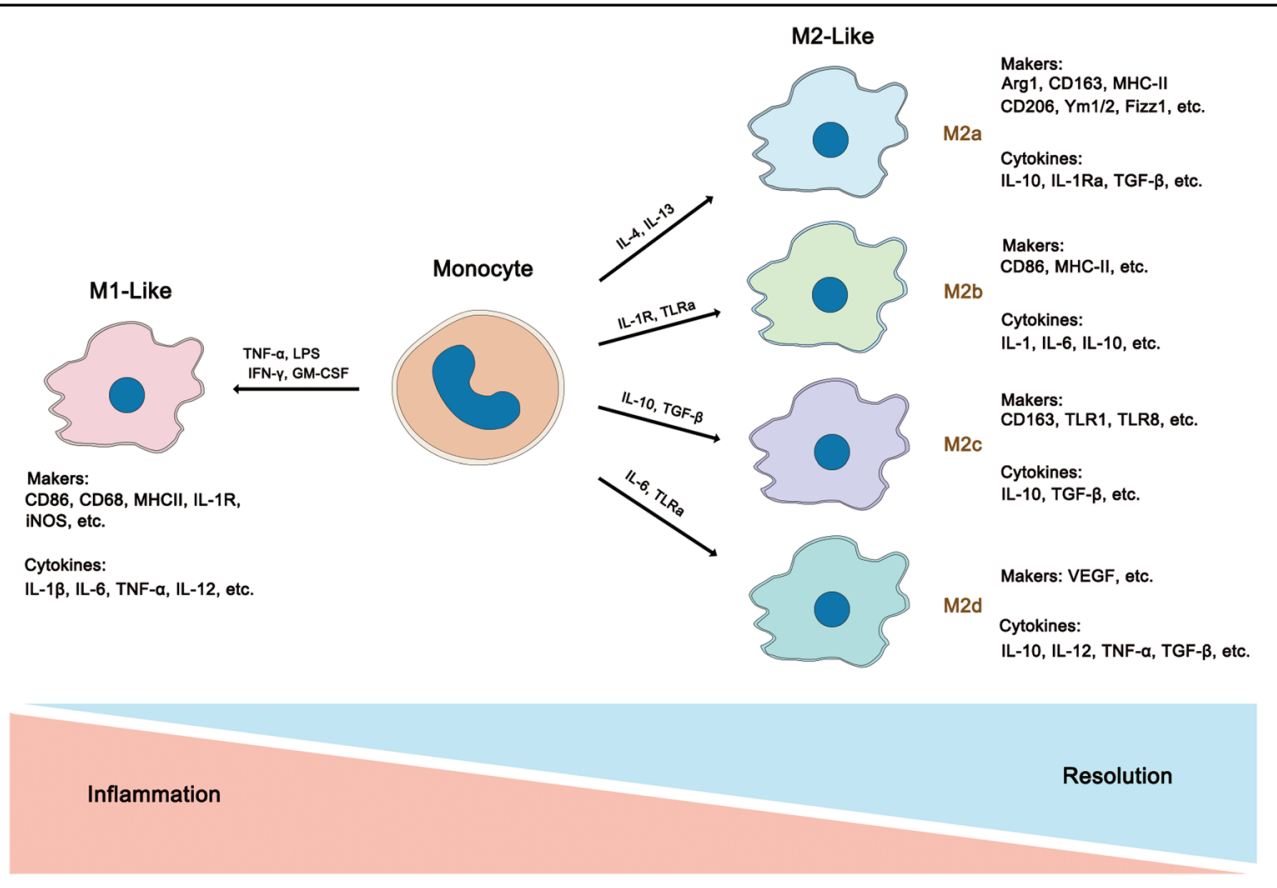

Fig. 1 The heterogeneity and characterizations of macrophages. Macrophages could be roughly divided into two subtypes (M1-like and M2-like, while M2-like macrophages can be further differentiated into M2a, M2b, M2c, and M2d phenotypes.) depending on their different microenvironmental stimuli. All of these phenotypes express different cytokines, chemokines, and receptors which give rise to their different functions respectively. Generally, M1-like macrophages mainly induce proinflammatory responses and usually associated with Th1 response while M2-like macrophages contribute trophism and tissue tolerance. Furthermore, M2a is mainly mediating tissue repair and remodeling and Th2 responses; M2b is commonly responsible for immunoregulation; M2c mainly functions in phagocytosis, and M2d participates in angiogenesis in tumor.

M2c macrophages are induced by IL-10, which express the Mer receptor tyrosine kinase and exhibit strong antiinflammatory and phagocytic effects ${ }^{14}$. M2d macrophages, also called tumor-associated macrophages (TAMs), belong to a newly identified branch of macrophages that are induced by TLR agonists and IL- $6^{12}$. M2like macrophages are identified by mannose receptor (MR/CD206), Arg1, IL-10, MHC class II molecules, peroxisome proliferator-activated receptor (PPAR $\gamma$ ), Fizz1 and YM1/2 family members.

In brief, M2 macrophages primarily participate in tissue repair, immunoregulation, and fibrosis. M2 macrophages often display lower capacity for antigen presentation and oxidants production and higher levels of certain antiinflammatory factors, such as IL-10 and TGF- $\beta$, thereby resolving deleterious inflammatory conditions ${ }^{15}$. However, hyper-activation of M2-like macrophages may induce tissue fibrosis, which is characterized by excessive extracellular matrix (ECM) deposition and destruction of normal tissue structure, resulting in organ dysfunction. In addition, M2-like macrophages also promote tumor progression and metastasis. TAMs are the most abundant immune cell population in tumors ${ }^{16}$, primarily originating from circulating monocytes and providing an immunosuppressive niche that supports tumor invasion.
Mechanistically, TAMs secrete matrix metalloproteinases (MMPs) and induce vascularization of tumor tissue by producing growth factors, such as vascular endothelial growth factor (VEGF), platelet derived growth factor (PDGF), and transforming growth factor (TGF)- $\beta^{17}$. Conversely, depletion or reprogramming of TAMs toward an M1-like phenotype has shown potential for cancer therapy ${ }^{18}$. Although TAMs are believed to be intimately associated with the anti-inflammatory TME, the traditional view of TAMs as skewed M2-like macrophages might be oversimplified. Since tumors are evolving tissues and molecules within TME and vary at different stages, the phenotypes of TAMs are dynamically altered in response to different TMEs ${ }^{19,20}$.

Unlike in vitro conditions, macrophages usually develop mixed phenotypes in vivo in response to disease conditions, and they are difficult to separate using classical M1 or M2 surface markers. For example, it has been reported that macrophage populations display a mixed M1/M2 phenotype in obese patients ${ }^{21}$. The dynamic balance between M1-like and M2-like macrophages tightly controls the disease outcomes, suggesting that regulation of macrophage phenotype is a promising strategy for disease treatment. In addition to cytokines release, increasing evidence indicates that EVs are also critical mediators for 
intercellular communication between macrophages and target cells or tissues during physiological and pathological processes. In the following sections, we discuss the biological properties of EVs.

\section{Properties of extracellular vesicles Diverse sub-populations of EVs}

Depending on their different origins and sizes, EVs were initially categorized into exosomes (Exos, $~ 30-200 \mathrm{~nm}$ ), microvesicles (MVs, $\sim 200-1000 \mathrm{~nm}$ ), and apoptotic bodies (ABs, $\sim 1-5 \mu \mathrm{m}$ ). However, with the increasing appreciations of EVs, many other sub-populations, such as exomeres $(<50 \mathrm{~nm})$ and large oncosomes (LOs, $\sim 1-10 \mu \mathrm{m}$ ), have been recently identified ${ }^{22}$. Briefly, ABs originate from the plasma membrane and are often produced when cells undergo apoptosis, exhibiting a broad size range between 1 and $5 \mu \mathrm{m}$ in diameter. Most ABs are engulfed by phagocytes through receptor recognition by factors such as Annexin V, C3b, and thrombospondin on their surface, which have served as well-accepted markers of apoptotic bodies ${ }^{23}$. Most apoptotic cells are eliminated by phagocytes in the form of ABs, a crucial biological process for avoiding autoimmunity ${ }^{24}$. Exomeres, first identified in 2018, are vesicles smaller than $50 \mathrm{~nm}$ lacking external membrane structures, and HSP90 has been proposed as a marker for exomeres. Exomeres contain abundant metabolic enzymes and signature proteins involved in the glycolysis and mTORC1 pathways ${ }^{25}$. However, detailed information and the biological function of exomeres remain largely unknown ${ }^{26}$. LOs are a large sub-populations of EVs $(\sim 1-10 \mu \mathrm{m})$ that are cancer cellspecific and are derived from the plasma membrane with high expression of ARF6 protein. Secretion of LOs is strongly associated with tumor aggressiveness ${ }^{27-29}$. However, it is unclear how much overlap in the markers and functions exists between LOs and other tumorderived EV sub-populations.

Despite much progress in the EV field, our understanding of the heterogeneity and diverse function of EV sub-populations is still in its infancy. Currently, the most common method to distinguish EV subtypes is dependent on their size ${ }^{30}$. However, due to a lack of specific markers, identification of any pure EV sub-population is not easy once they have been isolated in vitro. It is now believed that EVs are not only limited to these currently reported sub-populations ${ }^{22,31}$. More detailed and thorough investigations of EV populations are required, which will provide a better understanding of the diverse roles of EVs in disease pathology and treatment. In the literature related to macrophage-derived EVs, some subpopulations, such as exomeres, ABs, and LOs, are rarely involved. Therefore, we primarily focus on the biological roles of macrophage-derived Exos and MVs in the following sections.

\section{Regulation of EV biogenesis}

EVs are a group of nano-sized membranous vesicles released by live cells, that were initially considered to comprise a cell waste removal mechanism ${ }^{32}$. However, researchers have now recognized that EVs could carry and transfer biological molecules from parent cells to recipient cells, participating in intracellular communication during disease pathology and tissue regeneration $^{33}$. Based on their different origins and sizes, EVs are further categorized into at least two categories, exosomes (Exos, $\sim 30-200 \mathrm{~nm}$ ) and microvesicles (MVs, $\sim 200-1000 \mathrm{~nm})^{34,35}$. Briefly, EVs derived from the endosomal pathway are known as Exos and others derived from the plasma membrane are identified as MVs. Exos are primarily released by the fusion of plasma membrane (PM) and multivesicular bodies (MVBs) ${ }^{36}$. Internalization of $\mathrm{PM}$ produces early endosomes at the beginning, followed by invagination of endosomes that generates quantities of intraluminal vesicles (ILVs) within the endosomal compartments, leading to the formation of MVBs, which further fuse with the PM to release these ILVs to extracellular spaces, which then become Exos (Fig. 2). Although the detailed mechanism is not fully understand, it has been reported that the endosomal sorting complex required for transport (ESCRT) is intimately associated with the formation of Exos $^{37,38}$. However, knockdown of ESCRT allows the continued formation of Exos, suggesting that other mechanisms in addition to ESCRT are also involved in Exo biogenesis ${ }^{36,39,40}$. One of the alternative pathways involves synthesis of the sphingolipid ceramide ${ }^{41}$, which may have synergistic effects with ESCRT in the biogenesis of Exos. Another ESCRT-independent pathway is the family of tetraspanins, such as CD63, which plays important roles in the regulation of Exo biogenesis ${ }^{42-45}$. Furthermore, Rab proteins, which belong to the Ras-like small GTPase superfamily, have also been found to be associated with Exo release $e^{46,47}$.

MVs are thought to primarily originate from directly outward budding of the PM (Fig. 2). Despite their distinct origins, the membrane-trafficking process is involved in the formation of both MVs and Exos. Hence, they may share some common mechanisms of biogenesis. For example, ESCRT and Rab family have been reported to participate in MV formation as well ${ }^{46,48}$. Ras superfamily proteins, such as ADP-ribosylation factor 6 (ARF6), have also been proposed as key regulators of MV biogenesis ${ }^{49-}$ 51. ARF6 induces PM rearrangement, as well as alteration of actin dynamics and cytoskeleton which are involved in MV formation. In addition, high $\mathrm{Ca}^{2+}$ concentration promote $\mathrm{MV}$ release via reorganizing of the cytoskeleton $^{52}$. However, the specific process of MV biogenesis remains controversial, and additional studies are required to reveal the exact mechanism of MV biogenesis. 


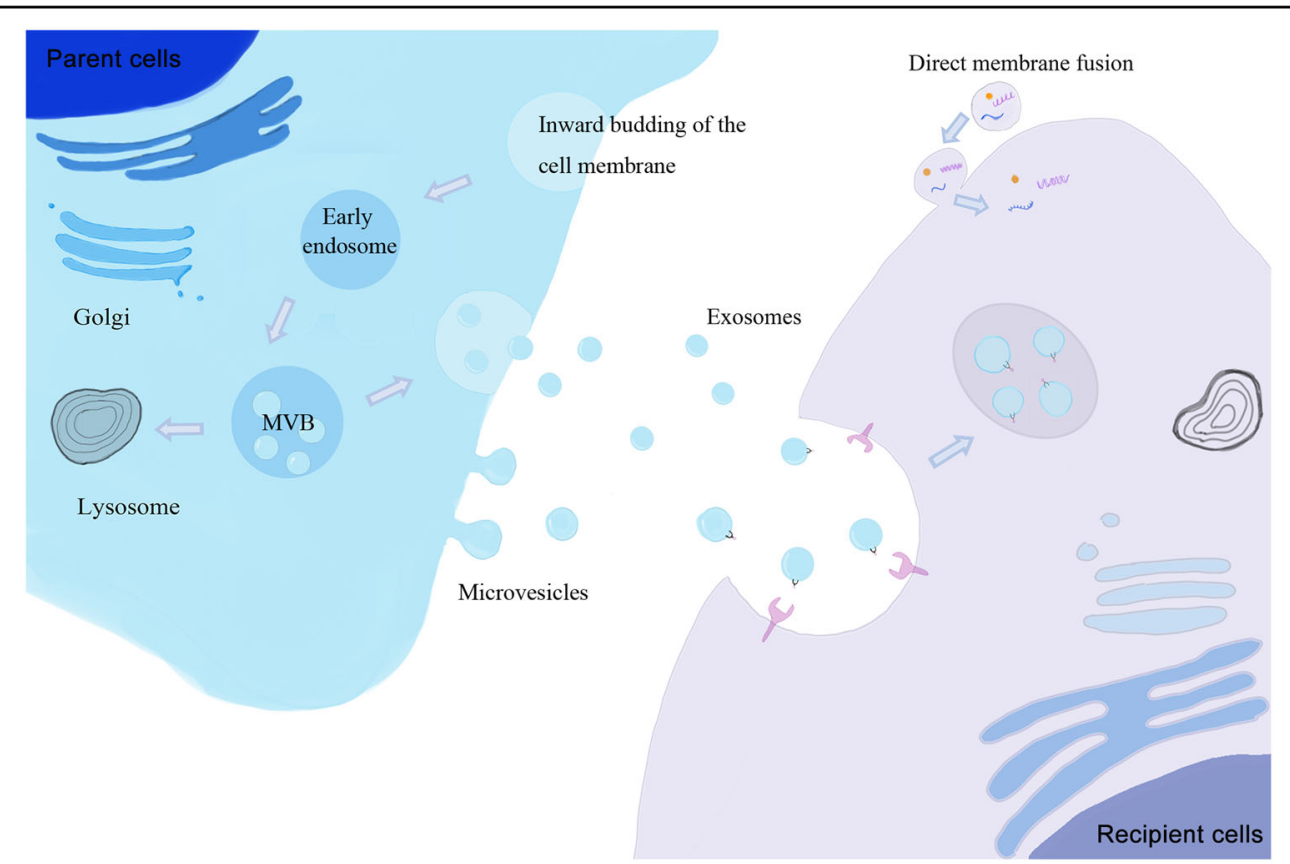

Fig. 2 Biogenesis and cellular uptake mechanisms of EVs. There are two major subtypes of EVs known as the Exos and the MVs. Exos are released by the fusion of plasma membrane with MVB which is generated by inward budding of endosome. While MVs are released by direct outward budding of plasma membrane. During cellular uptake, contents within released EVs from parent cells can be transmitted into the cytoplasm of receptor cells by membrane fusion and/or endocytosis. In this endocytosis process, clathrin-mediated endocytosis (CME), macropinocytosis, and phagocytosis are regarded as the most common pathways.

\section{Cellular uptake mechanism of EVs}

EVs can encase multiple types of bioactive molecules such as proteins, RNAs (e.g., mRNAs, miRNAs, mtRNAs, and lncRNAs), DNAs (e.g., mtDNA), and lipids from parent cells. Released EVs are further taken up by recipient cells and regulate gene expression or signaling pathways in these cells. Several mechanisms, such as protein interactions, endocytosis, and direct membrane fusion, have been implicated in the cellular uptake process of EVs (Fig. 2). Protein interactions between EVs and recipient cells may promote subsequent endocytosis. After treatment with Proteinase $\mathrm{K}$ to degrade proteins, EVs exhibited a dramatic decrease in cellular uptake rates, suggesting the important roles of protein interactions in this process ${ }^{53}$. In addition, uptake of EVs by endocytosis seems to be the most common pathways, including clathrin-mediated endocytosis (CME), macropinocytosis, and phagocytosis. The cellular uptake of EVs through the CME pathway depends extensively on cholesterol that is enriched in lipid rafts ${ }^{54}$. Flotillins is a common marker protein of lipid rafts that has intimate associations with endocytosis in a CME independent pathway. Another proposed mechanism is the direct membrane fusion of EVs and recipient cells, and several proteins, such as Rab and SNAREs, participate in this process ${ }^{55}$. Moreover, acidic $\mathrm{pH}$ conditions, such as in tumors and the stomach, also promote membrane fusion of EVs with target cells ${ }^{56}$.
Although several mechanisms have been proposed, it seems that the cellular uptake of EVs involves more than one single mechanism, and multiple pathways work together simultaneously to modulate this process.

\section{EV isolation and characteristic methods}

Isolation of EVs is the first step that directly determines the quality of subsequent research. Currently, several EV isolation methods have been established, and each method has its own advantages and disadvantages. Ultracentrifugation (UC) is the most common isolation method, which obtain EVs based on differential centrifugal force ${ }^{57}$. In brief, liquid samples go through a series of centrifugation to remove cells, debris, apoptotic bodies, and finally collect EVs by UC. UC is widely used in EV isolation due to its high suitability for multifarious liquid samples and simplicity compared to other methods. However, damage to the EVs structure caused by UC is irreversible and may influence subsequent tests. Contamination by aggregate proteins is also a considerable issue ${ }^{57}$. Density gradient flotation is often applied to improve the purity of EVs depending on their density, which is performed through a gradually increasing density to isolate EVs. However, both UC and density gradient flotation are time-confusing and laborintensive. Other methods including ultrafiltration, sizeexclusion chromatography (SEC), immunoaffinity, and precipitation are also applied in EV isolation. Ultrafiltration 
and SEC are both based on sizes. Although ultrafiltration is time-saving compared to UC, low yield largely impedes its application. EV isolation by SEC is believed to have high purity and integrity compared to other methods. However, elution in SEC may reduce EVs concentration and influence EVs functions ${ }^{58,59}$. Although immunoaffinity exhibits high purity and isolates EVs through specific antibodies that recognize surface markers on EVs, it unsuitable for large volume samples. Recently, several commercial isolation kits have been established that are largely based on the precipitation of EVs in polymer solution. While the high output and convenient process have aroused interest, impurity with lipoproteins and introduced polymers during precipitation has raised some concerns ${ }^{60}$.

Recently, newer EV isolation and detection methods have also been developed. For instance, microfluidic platforms, including size-based separation, immunoaffinity-based separation, and dynamic separation, are primarily based on the size and immunoaffinity of EVs. Despite some advantages of microfluidics, such as high purity, cost-effciency, and portable properties, shortcomings, such as complicated photolithography fabrication and capturing EVs with only targeted proteins, also exist. Furthermore, nanoscale flow cytometry (nanoFACS) has been used to analyze and sort EV sub-populations using specific antibodies against EV marker proteins ${ }^{61,62}$. Due to its highresolution, nanoFACS obliterates noise and background and determines the cellular source of EVs, which has made it extremely valuable ${ }^{63}$. Asymmetrical flow field flow fractionation (AF4) is another isolation method that separates EVs based on their different sizes ${ }^{25,64}$. AF4 technology is coupled with a multidetection system composed of ultraviolet-visible spectroscopy (UV) and multiangle light scattering (MALS), which separates and characterizes EVs according to their different diffusion coefficients, since particle size positively correlates with its diffusion coefficient. Although many novel tools have been developed, there is still no current standard for EV isolation or analytic methods, with each method having its own strengths and weaknesses. Therefore, it is necessary to select an appropriate EV isolation method based on the purpose of study.

Characterization of EVs primarily involves morphological and molecular identification. Transmission electron microscopy (TEM) and nanoparticle tracking analysis (NTA) are commonly employed to detect the complete spherical structures and nano-scales of EVs respectively. Western blotting is usually performed to detect EV protein markers, such as TSG101, CD63, ALIX, etc. Notably, none of these methods alone can characterize EVs, and EV characterization requires several methods used collectively ${ }^{36,65}$.

\section{Pathological roles of $M \varphi-E V s$ in disease}

Macrophages-derived EVs (M $\phi$-EVs) deliver abundant proteins, lipids, and genetic information among cells to modify the phenotype and function of target cells. However, contents of $\mathrm{M} \phi$-EVs may vary with different $\mathrm{M} \phi$ phenotypes or microenvironments. For example, EVs from polarized and naïve macrophages display distinct miRNA profiles ${ }^{66}$. Since macrophages usually develop complicated and mixed phenotypes in response to different diseases or different phases even in the same disease in vivo, it is not easy to identify the exact subsets (e.g., M1 or M2) of their EVs. Hence, we discuss the roles of macrophages-associated EVs in the pathology of different diseases in the following sections (Table 1 and Figs. 3 and 4).

\section{Cardiovascular diseases}

Cardiovascular diseases (CVDs), such as coronary heart disease, peripheral arterial disease, and cerebrovascular disease, are a class of disorders of heart and blood vessels. Macrophages have been recognized as vital players that participated in the most stages of CVDs. For example, in atherosclerosis, macrophages within plaques scavenge retained lipoproteins and transform into cholesterol-laden foam cells, which subsequently exacerbate inflammatory responses and accelerate atherosclerosis progression ${ }^{67,68}$. Although the detailed mechanisms remain elusively, recent studies have realized the important roles of $\mathrm{M} \phi$ EVs in the development of CVDs. For example, EVs from atherogenic macrophages promote plaque formation in atherosclerosis. These EVs restrained the migration of naïve macrophage out of plaques and reduced the growth and tube formation of endothelial cells (ECs), increasing plague progression ${ }^{69,70}$. Moreover, excessive lipoproteins accumulation induces the release of $\mathrm{M} \phi$-EVs enriched with lncRNA GAS5, triggering apoptosis in $\mathrm{ECs}^{71}$. Vascular smooth muscle cells (VSMCs) usually reside close to macrophage infiltration sites during vascular lesions ${ }^{72}$. Recently, EVs from M1-like macrophages have been proven to activate extracellular regulated protein kinase (ERK) and protein kinase B (Akt) pathway by transferring integrins, which in turn stimulate extracellular matrix (ECM) production and cell migration and adhesion in VSMCs, aggravating atherosclerosis ${ }^{73}$. Additionally, EVs from macrophages exposed to nicotine also induce VSMC proliferation and migration by delivering miR-21-3p to activate phosphatase and tension homolog (PTEN) in these cells ${ }^{74}$.

Cardiac remodeling is a subsequent pathological condition following severe CVDs. Although the detailed mechanism is still under investigation, macrophage infiltration has been regarded as one of the vital factors in this course $^{75,76}$. Macrophage-derived EVs are taken up by cardiac fibroblasts, and a macrophage-fibroblast crosstalk has been proposed. After myocardial infarction (MI), EVs derived from proinflammatory macrophages deliver miR155 into cardiac fibroblasts, inhibiting their proliferation, 


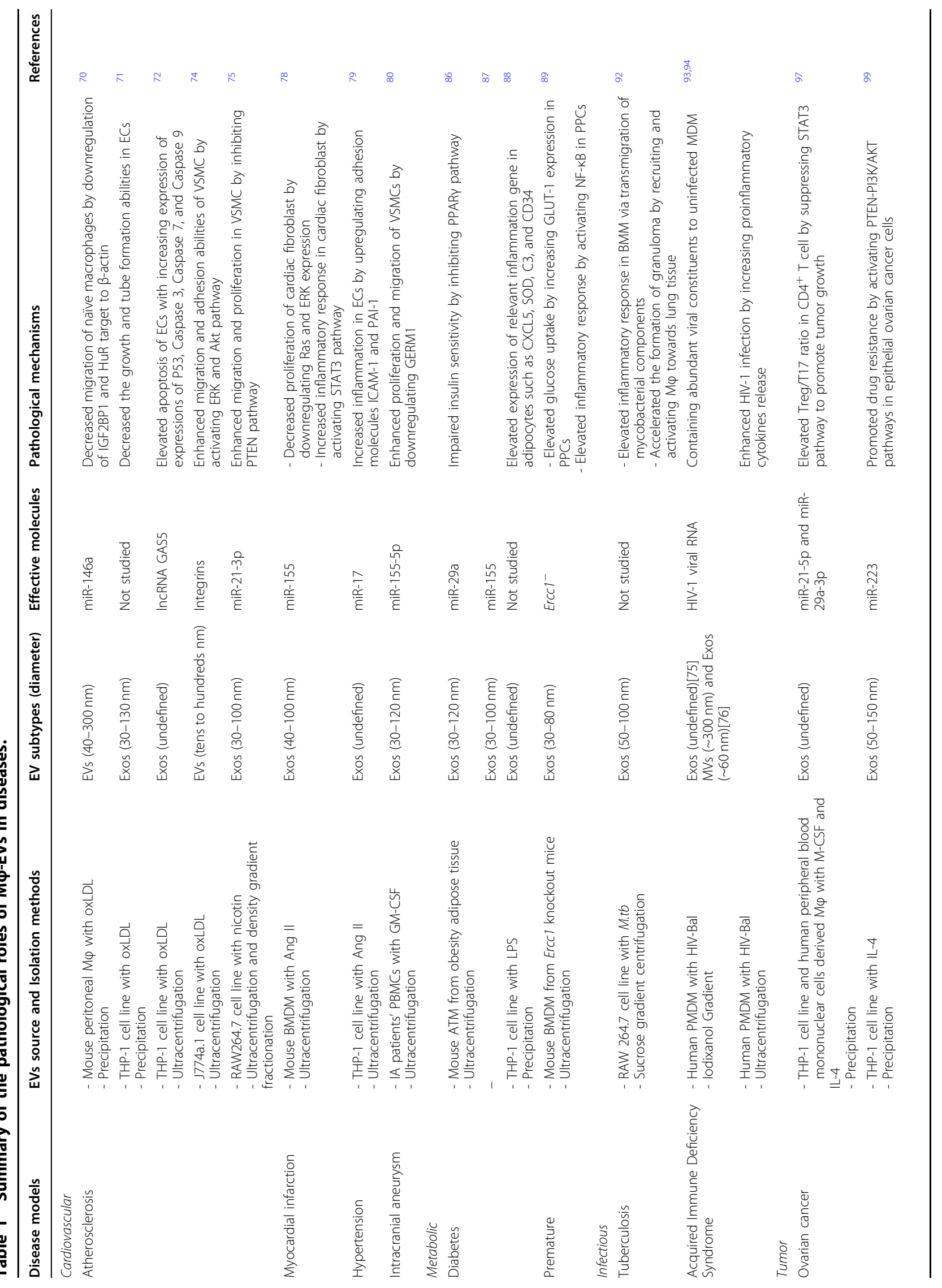




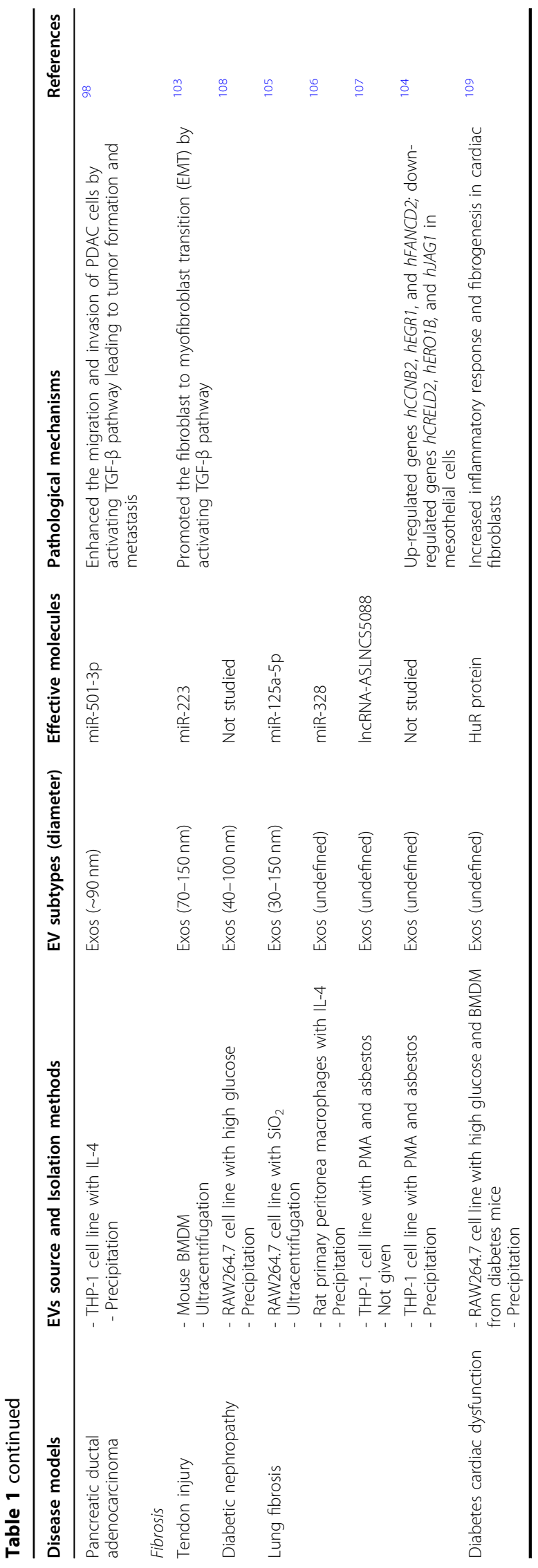

causing abnormal cardiac remodeling and increasing the incidence of cardiac rupture ${ }^{77}$. Furthermore, EVs isolated from macrophages under hypertension were enriched with miR-17 and induce the expression of cytokines, such as intercellular adhesion molecule-1 (ICAM-1) and plasminogen activator inhibitor-1 (PAI-1), in ECs via activating the NF- $\mathrm{kB}$ pathway, which ultimately promote cardiac inflammation and pathological cardiovascular remodeling ${ }^{78}$. During intracranial aneurysm (IA) development, $M \phi$-EVs transfer miR-155-5p to promote the proliferation and migration of smooth muscle cells by downregulating GREM1, a member of the antagonists of secreted bone morphogenetic protein ${ }^{79}$. Collectively, although the relevant mechanisms are still under debate, the considerable effects of M $\phi$-EVs during CVDs are indispensable to disease development.

\section{Metabolic diseases}

Metabolic diseases, such as obesity, insulin resistance (IR), and diabetes, belong to a type of complicated metabolic disorders with both high morbidity and mortality $^{80,81}$. Macrophages-mediated inflammatory responses are recognized as an essential pathological process to disease progression. For example, increased numbers of adipose tissue macrophages (ATMs) have been observed in IR individuals ${ }^{82}$ and the morbidity of obesity and IR strongly parallels with the severity of inflammation ${ }^{83}$. Moreover, co-culture of ATMs with pancreatic cells demonstrated that cell-cell communication is responsible for inflammatory milieu formation ${ }^{84}$. EVs from obese ATMs induce glucose intolerance and IR, while these adverse effects could be reversed by EVs from lean $\mathrm{ATMs}^{85}$. PPAR $\gamma$ is a key factor for maintaining insulin sensitivity, and EVs from proinflammatory macrophages induce glucose intolerance and IR by disrupting PPAR $\gamma$ signaling. EVs isolated from obesity mice-derived ATMs deliver miR-155 and miR-29a to inhibit PPARY in adipocytes, myocytes, and hepatocytes, increasing levels of fasting blood glucose, serum insulin, and index of homeostasis model assessment of IR in lean mice ${ }^{85,86}$. However, another study reported that EVs from LPSinduced macrophages failed to influence glucose uptake and fat storage in adipocytes but were able to up-regulate inflammation and carbohydrate catabolism related genes such as CXCL5, SOD, C3, and CD34 in adipocytes ${ }^{87}$. Additionally, macrophages derived from mice with knockout of Ercc1, a highly conserved endonuclease complex required for lesion excision in nucleotide excision repair, release EVs to induce glucose transporter type-1 (GLUT-1) expression in pancreatic cells, further leading to hyperglycemia and inflammation in primary pancreatic cells ${ }^{88}$. Overall, these findings collectively verified that $\mathrm{M} \phi-\mathrm{EVs}$ promote metabolic diseases 


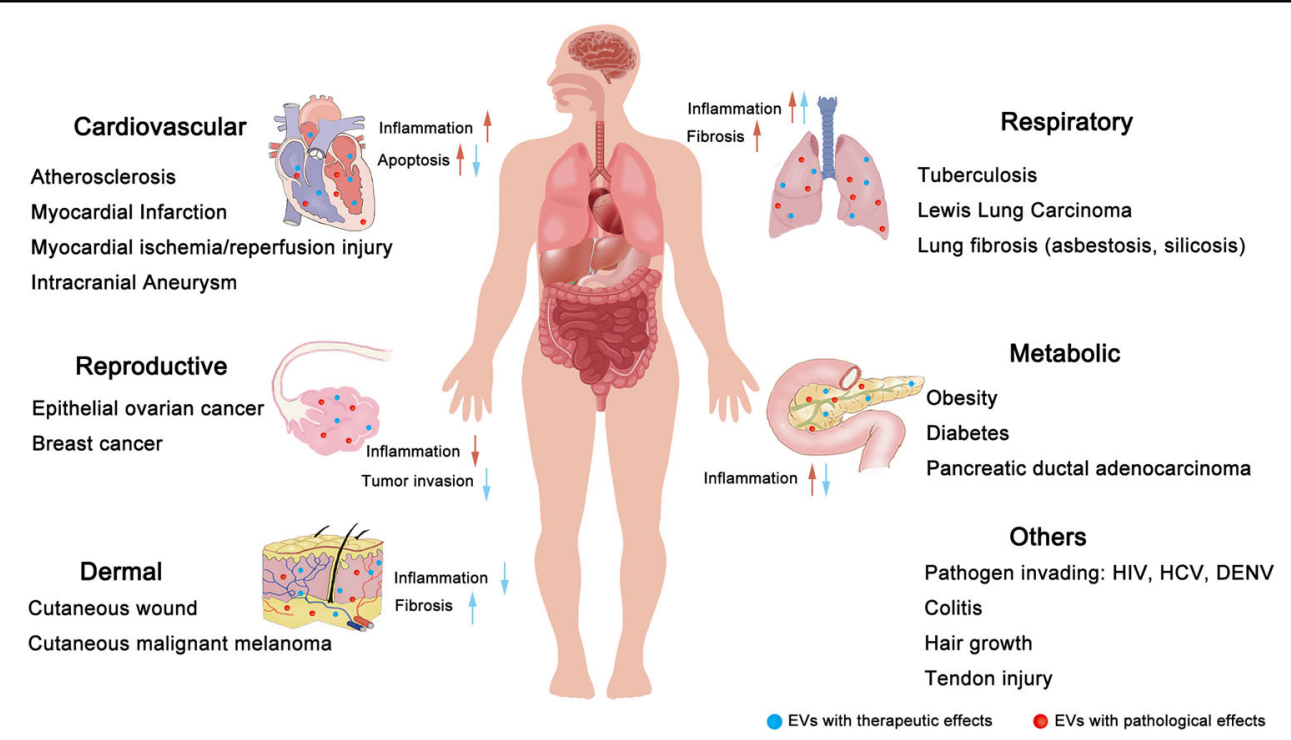

Fig. 3 Summary of the current findings for various diseases associated with macrophages-derived EVs. EVs derived from macrophages contain different types of constitutes that can affect the functions of multifarious systems in vivo. Specifically, the red arrows imply for the pathological roles of EVs in diseases and the blue ones imply for their therapeutic roles.

development by modulating glucose and lipid metabolism pathways.

\section{Infectious diseases}

The key roles of macrophages against foreign invaders have been known for years. Investigators have recently realized that $\mathrm{M} \phi$-EVs also exert several adverse roles during severe infections. Mycobacterium tuberculosis $(M$. $t b$ )-induced tuberculosis (TB) is characterized by a series of severe inflammation responses along with the formation of granuloma and drug-resistance ${ }^{89}$. Several studies suggested that EVs from M.tb infected macrophages contribute to TB development. M.tb infected macrophages package mycobacterial lipids and proteins within their EVs, and these EVs further induce the formation of granuloma and facilitate M.tb spread $^{90,91}$. Furthermore, $\mathrm{M} \phi$-EVs are also implicated in viral infections such as human immunodeficiency virus (HIV) and hepatitis C virus $(\mathrm{HCV})$. An increasing number of EVs has been found in HIV-infected monocyte-derived macrophages (MDM). Despite these EVs differing in size, morphology, and content (miRNA and proteomic profiles), they were able to deliver viral constituents to the uninfected MDM and thus induce infection in these cells ${ }^{92,93}$. Epidemiological data indicate that individuals infected with HIV are at high risk for the development of pulmonary diseases, but the underlying mechanism whereby this occurs remains unclear $^{94}$. Interestingly, HIV-infected macrophages were found to release EVs plentiful in miR-130a, which induces pulmonary smooth muscle cell proliferation and development of pulmonary arterial hypertension ${ }^{92}$. Moreover, EVs from HIV-infected macrophages are rich in miR-23a and miR-27a, which damaged the EC integrity and caused mitochondrial dysfunction, ultimately exacerbating pulmonary lesions ${ }^{93}$. These findings suggest that EVs from infected macrophages can transfer the pathogenic constitutes or miRNAs to aggravate pathogen spread or disease severity. However, the ability of EVs to carry pathogenic constituents may also shed light on their application for pathogen vaccines.

\section{Tumor development and chemoresistance}

Throughout the tumor microenvironment (TME), multiple cell type such as immune cells, vascular endothelial cells, and fibroblasts collectively work to regulate tumorigenesis. Disorders of the TME increase the occurrence of tumor recurrence, metastasis, and chemoresistance ${ }^{95}$. TAMs are one of the major TME cells that provide an immunosuppressive milieu for tumor progression and secrete many growth factors that suppress the proinflammatory cytokine release in the TME. It is thought that TAM-derived EVs (TAM-EVs) exhibit similar tumorfriendly properties to their donor cells. These EVs have shown the capacity to mediate cell-to-cell communication between TAMs and other immune cells such as T cells. For example, TAM-EVs enriched with miR-21-5p and miR29a-3p inhibit the STAT3 pathway and induce Treg/Th17 cell imbalance, generating an immunosuppressive milieu to facilitate the progression and metastasis of epithelial ovarian cancer $^{96}$. TAM-EVs also deliver miR-501 to activate the TGF- $\beta$ pathway and promote tumor migration and invasion 


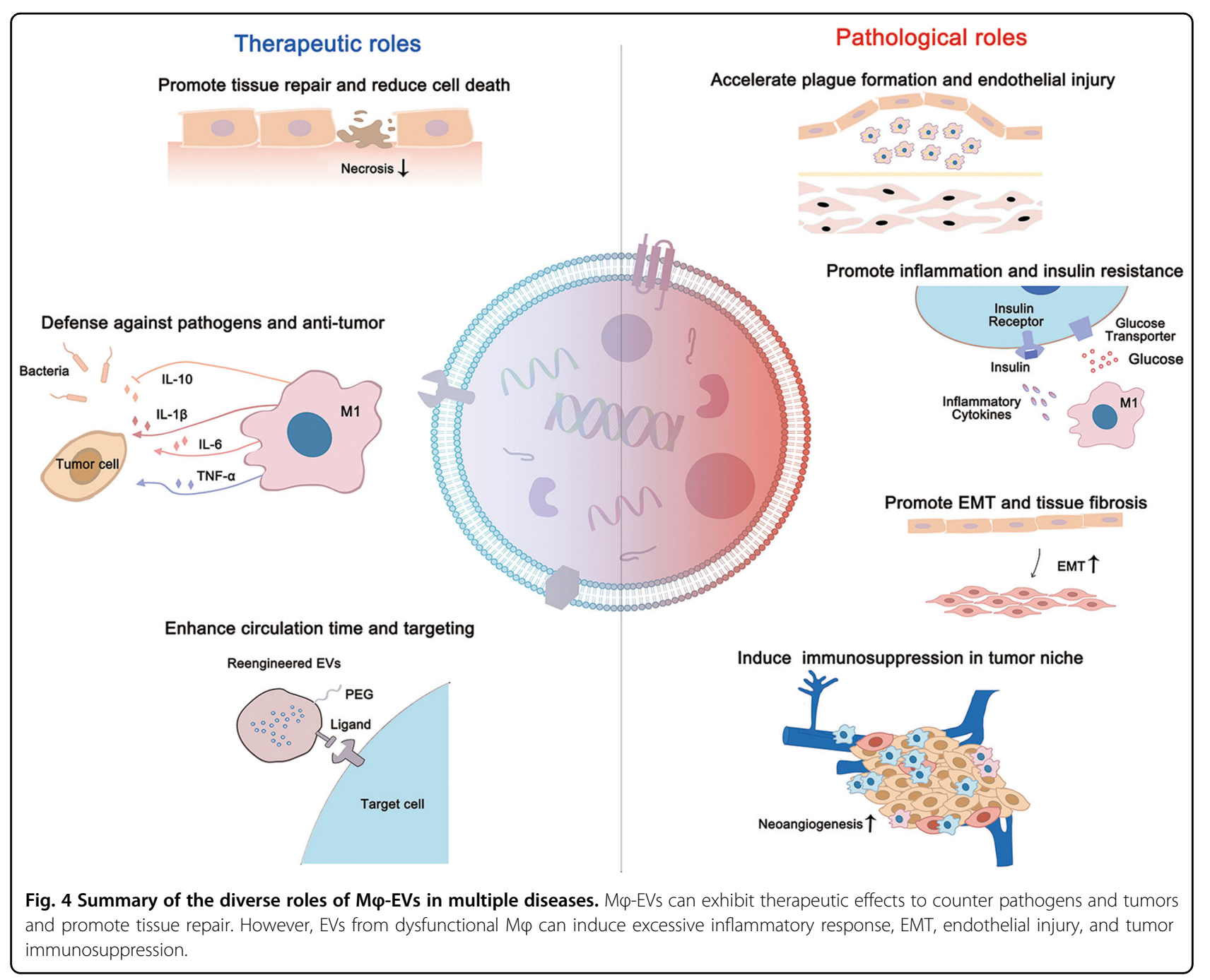

in pancreatic ductal adenocarcinoma ${ }^{97}$. Moreover, TAMEVs play important roles in the pathology of tumor chemoresistance. It has been reported that EVs from TAMs under hypoxic conditions with enriched miR-223 promote drug resistance in epithelial ovarian cancer through activating PTEN-PI3K/AKT pathways ${ }^{98}$. However, another study argued that EVs derived from TAMs display molecular profiles associated with Th1/M1 polarization, and they might exhibit antitumor immunity ${ }^{99}$. Taken together, TAM-EVs have emerged as vital mediators in tumors development and chemoresistance, and their detailed effects in tumors still need to be investigated in future studies.

\section{Tissue fibrosis}

Tissue fibrosis is characterized as the up-regulation of profibrotic factors (e.g., TGF- $\beta$, CTGF) and ECM components (e.g., Fibronectin, Collagen type I) that destroys normal histological structures, ultimately leading to organ dysfunction $^{100,101}$. Recently, M $\phi$-EVs have been implicated in the pathology of tissue fibrosis, as their administration restarts the profibrotic pathways after depletion of macrophages in mice ${ }^{102}$. Asbestos exposure is a major cause of several severe lung diseases such as malignant mesothelioma (MM), lung fibrosis (asbestosis), and bronchial carcinoma. Evidence has shown that EVs from asbestos exposed macrophages induce the expression of many genes that are involved in TGF- $\beta$-meditated cell-cycle control and chromosome instability in mesothelial cells, contributing to the development of lung fibrosis ${ }^{103}$. Lung silicosis induced by silica particles is primarily characterized by diffuse fibrosis. EVs from macrophages exposed to silica also up-regulate $\alpha$-SMA expression in fibroblasts due to the abundant miR-125a$5 \mathrm{p}$ in these EVs ${ }^{104}$. Moreover, EVs from M2-like macrophages are enriched with miR-328 and IncRNAASLNCS5088, which subsequently activates TGF- $\beta$ and profibrotic factors (e.g., $\alpha$-SMA, Collagen I) in fibroblasts ${ }^{105,106}$.

Progressive renal fibrosis is a hallmark of diabetic nephropathy (DN), a leading cause of end-stage renal 
disease (ESRD) and renal failure in huge number of diabetic patients. Although macrophage infiltration is linked to the progression of $\mathrm{DN}$, their roles in renal fibrosis during $\mathrm{DN}$ are not entirely clear. Interestingly, a recent study found that EVs from macrophages under high glucose condition were taken up by mesangial cells, enhancing the proliferation and excessive ECM secretion in these cells via activating TGF- $\beta 1 /$ Smad3 pathways ${ }^{107}$. $\mathrm{M} \phi$-EVs from diabetic conditions were abundant with $\mathrm{HuR}$, an RNA-binding protein that regulates post-transcription, and induce fibrogenesis in fibroblasts ${ }^{108}$. As a result, angiotensin II-induced CVD mice supplemented with EVs carrying HuR displayed clear interstitial and perivascular fibrosis ${ }^{108}$. Collectively, these findings have revealed the significant roles of EVs in the pathology of tissue fibrosis in multiple organs.

\section{Therapeutic roles of $M \varphi$-EVs in diseases}

Depending on their parental cell types and the cargos they carry, M $\phi$-EVs may exhibit either immunosuppressive or immunostimulatory effects, thus serving as potential tools for disease therapy. As a type of endogenous nanovesicles, EVs have several advantages such as low immunogenicity, nontoxicity, and higher stability, over other synthetic nanoparticles ${ }^{109}$. Moreover, EVs exhibit the ability to pass through tissue barriers, such as the $\mathrm{BBB}^{110}$, enhancing the therapeutic efficacy of incorporated molecules to target cells. M $\phi$-EVs have emerged as a promising cell-free therapy for many biomedical applications such as tissue repair, drug delivery, and pathogen control (Table 2 and Figs. 3 and 4).

\section{Tissue repair}

Functional recovery of injured tissues remains a huge clinical challenge, and $M \phi$-EVs have shown several beneficial effects on injured cells or tissues. For example, myocardial ischemia/reperfusion ( $/$ R) injury is characterized by irreversible injury to the myocardium and results in heart dysfunction. Interestingly, EVs from M2like macrophages deliver miR-148a to mitigate I/Rinduced myocardial injury via suppressing the overloaded $\mathrm{Ca}^{2+}$ and inflammatory cytokine production in cardiomyocytes ${ }^{111}$. The anti-inflammatory effect of $M \phi$-EVs was also investigated in the models of diabetic wound, which is characterized by a persistent inflammatory response. For example, the skin defect in diabetic rats was rescued by EVs derived from anti-inflammatory macrophages ${ }^{112}$. Mechanistically, these EVs not only reduced cytokine (e.g., TNF- $\alpha$, IL-6) secretion in ECs, but also induced EC proliferation and migration to improve angiogenesis and re-epithelialization during wound healing ${ }^{112}$. Furthermore, $\mathrm{M} \phi$-EVs promote hair growth and protect the intestine against radiation injury through activation of $\mathrm{WNT} / \beta$-catenin signaling ${ }^{113,114}$.
$\mathrm{M} \phi$-EV-guided immune cell reprogramming is a promising therapeutic approach for inflammation-associated disorders. EVs from M2b macrophages display a more robust protective capacity compared to those from M2a macrophages or M2c macrophages in dextran sulfate sodium-induced colitis. The anti-inflammatory effects of M2b-derived EVs may be ascribed to elevated CCL1, which promotes Th2 polarization of the colon to rebalance the Th1 immune response, thereby attenuating the severity of DSS-induced colitis in mice ${ }^{115}$. M $\phi$-EVs also induce phenotype switching in macrophages, and subcutaneous injection of M2-like macrophages-derived EVs in a cutaneous wound induced local macrophage phenotype switching from M1-like towards M2-like, promoting the cutaneous wound healing by enhancing angiogenesis, re-epithelialization, and collagen deposition ${ }^{116}$.

\section{Pathogen control}

Macrophages are one type of primary innate immune cells that can fight pathogen infection. The therapeutic role of $\mathrm{M} \phi$-EVs also sheds light on infectious diseases. For instance, EVs from M.tb-infected macrophages are capable of inducing the systematic inflammatory responses with high levels of cytokines (e.g., TNF- $\alpha$, IL-12) in primary macrophages and M.tb-infected mice, which was indispensable at the early phase of pathogen control ${ }^{91,117}$. Similarly, EVs derived from M.tb-infected macrophages transfer M.tb RNA into naïve macrophages and activate the intracellular RNA sensing pathway, promoting Th1 immune response with the release of IFNs ${ }^{118}$. In addition, $\mathrm{M} \phi$-EVs carry viral proteins to irritate the immune system during infections. It has been found that EVs from M.tbinfected macrophages carried mycobacterial proteins, and these EV-vaccinated mice exhibited a robust Th1 response with limited Th2 response, suggesting that $M \varphi-$ EVs may be regarded as promising cell-free agents against $\mathrm{TB}^{119,120}$. During the early stage of fungal infection, EV contents from Candida albicans-infected macrophages were extensively altered and were intimately associated with enhanced cytokine secretion in macrophages ${ }^{121}$. Moreover, EVs from dengue virus (DENV)-infected macrophages also transfer the non-structural protein (NS3) encoded by DENV RNA to promote cytokine release in ECs, activating the defense program against dengue virus infection at the early stage ${ }^{122}$. EVs released from toll-like receptor 3 (TLR3)-activated macrophages, which are often blocked during infection, were found to possess abundant miR-29 to inhibit HCV replication in hepatocytes ${ }^{123}$. Interestingly, it seems that macrophages may have certain mechanisms to mediate the release pattern and synergistic effects of cytokines and EVs in response to infections. For example, macrophages orchestrate a fast but short-lasting antiviral state by secreting cytokines during HCV infection, while their EVs 


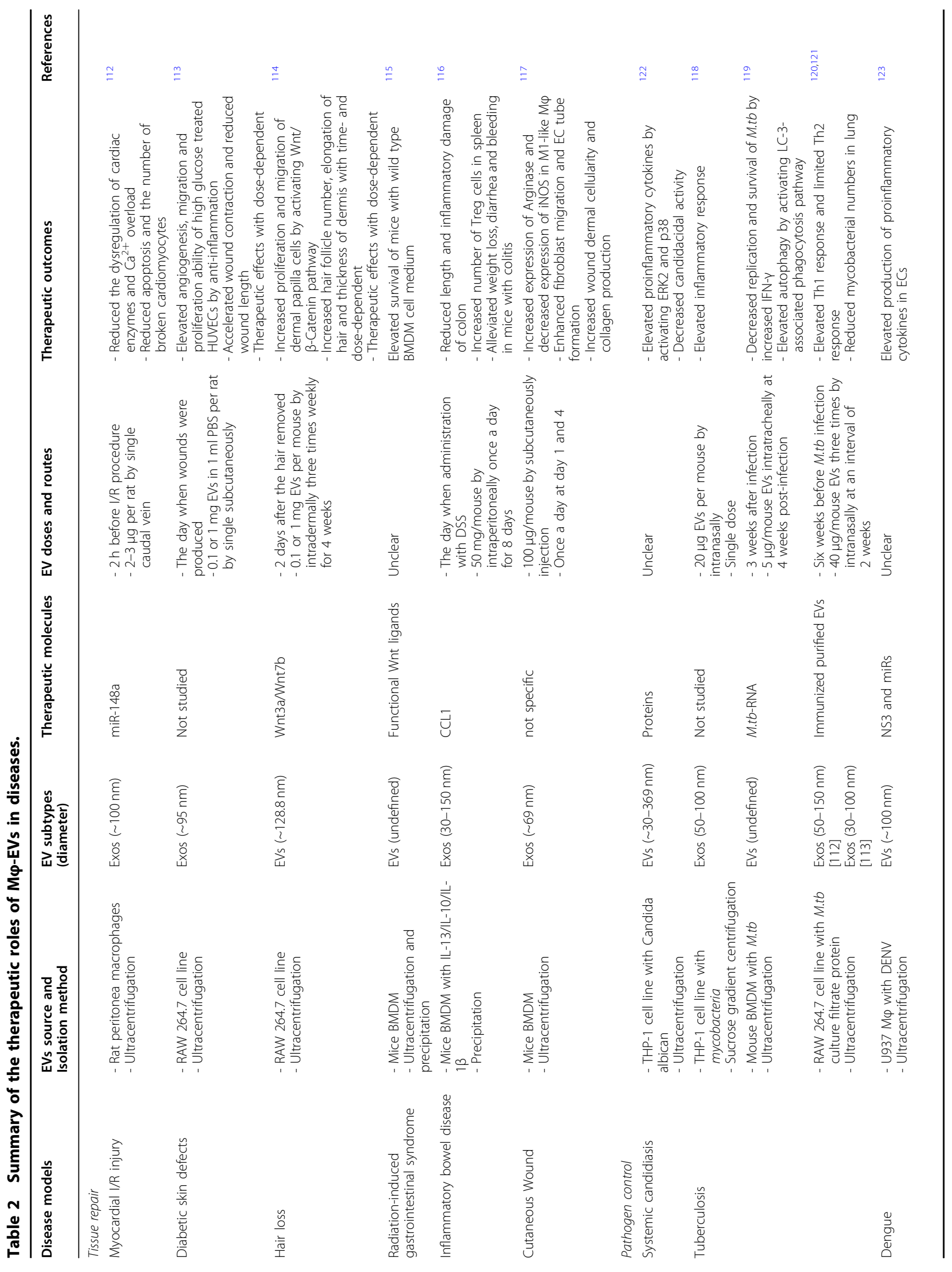




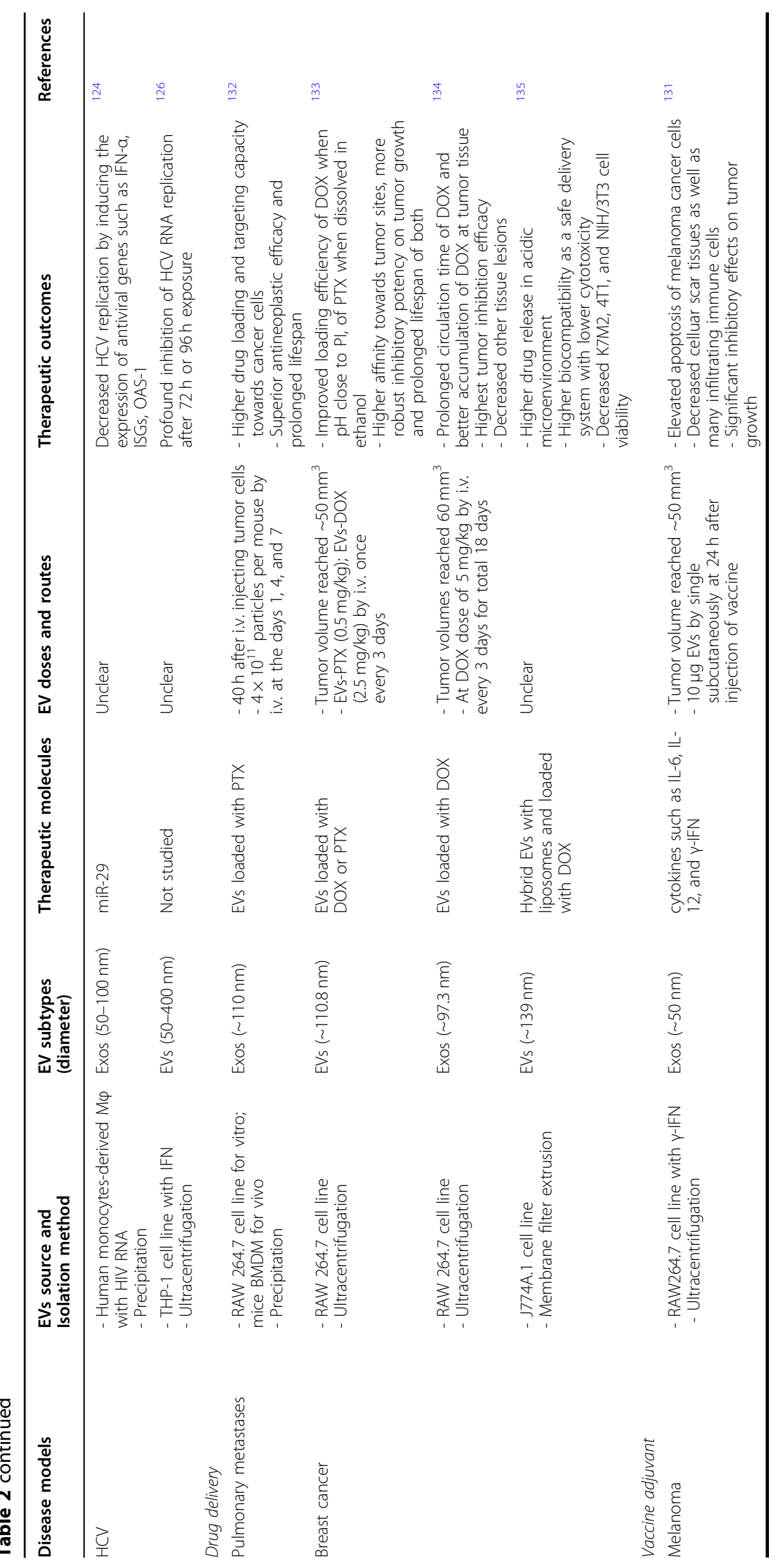


induce a late but long-lasting inhibition on HCV replication in hepatocytes with sub-genomic HCV replicons $^{124}$. These studies suggest that $\mathrm{M} \phi-E V s$ may boost the anti-infective immune response by delivering viral materials or therapeutic molecules.

\section{Drug delivery}

EVs have lipid bilayer membrane structures, allowing them to encapsulate and deliver various types of bioactive molecules in vivo ${ }^{36,125}$. The nanoscale size and natural bilipid membrane of EVs allow them to easily pass through biological barriers (e.g., blood-brain barrier) ${ }^{126,127}$. Moreover, EVs from macrophages express CD47, a surface molecule known as the "don't eat me" signal to escape immunological surveillance ${ }^{128,129}$. Therefore, application of M $\phi$-EVs as drug delivery vehicles has received considerable attraction. EVs from M1like macrophages alone showed a mild antitumor effect $^{97,130}$, but they were capable of restricting tumor growth when combined with other therapeutic agents. For example, M $\phi$-EVs loaded with Paclitaxel (PTX) exhibited a robust accumulation in Lewis lung carcinoma (LLC) cells both in vitro and in vivo. The EV-PTX combination significantly reduced lung metastases and prolonged lifespan in an LLC metastasis mouse model ${ }^{131}$. Although $\mathrm{M} \phi$-EVs have shown potential for cancer therapy, their poor drug loading efficacy largely impedes further clinical translation. Thus, optimized drug loading processes for $\mathrm{M} \phi$-EVs have also been studied. For example, the loading efficiency of M $\phi$-EVs with Doxorubicin (Dox) was improved when $\mathrm{pH}$ was close to the $\mathrm{pI}$ of Dox, and PTX dissolved in ethanol was efficiently encapsulated into $\mathrm{M} \phi-$ EVs. Accordingly, these optimized drug-loaded M $\phi$-EVs displayed higher inhibitory potency on tumor growth in orthotopic triple-negative breast cancer (TNBC) tumor models compared to the original drug-loaded EVs or drug alone $\mathrm{e}^{132}$.

In addition, some hybrid strategies have been proposed to enhance the therapeutic potency of $\mathrm{M} \phi-\mathrm{EV}$ s. For example, TNBC is aggressive and often returns after treatment. To resolve this issue, $\mathrm{M} \phi$-EVs were engineered by removing their own contents and modifying addition of c-Met onto their surface, after which Dox-loaded poly lactic-co-glycolic acid (PLGA) nanoparticles were wrapped into the empty $M \phi$-EVs. These engineered EVs prolonged the release profile of Dox in vitro, and displayed high tumor targeting ability and excellent tumor inhibitory efficiency in mice with $\mathrm{TNBC}^{133}$. Another example is hybridizing EVs from M1-like macrophages with liposomes: the resulting hybrid M $\phi$-EVs exhibited higher cytotoxicity to multiple types of cancer cells, such as osteosarcoma and breast cancer cells, when loaded with $\operatorname{Dox}^{134}$. These studies indicate that M $\phi$-EVs constitute a promising natural carrier for target drug delivery.
However, despite these efforts, obstacles are still substantively unresolved with respect to obtaining EVs with the desires properties.

\section{Adjuvant cancer vaccines}

Cancer immunotherapy functions are primarily based on the patient's own immune system, and it is believed to be an important shift in tumor therapy. Cancer vaccines are commonly needed in combination with other therapies or adjuvants to improve the magnitude, breadth, quality, and longevity of the immune response to vaccines $^{135}$. Although great progress has been made recent years, some limitations still exist in many adjuvants, such as induction of disabled immune responses. Recently, EVs derived from the proinflammatory macrophages have been proposed as promising adjuvants. Exogenous supplementation with EVs from M1-like macrophages skewed naïve macrophages towards a proinflammatory state within $4 \mathrm{~h}$, generating a proinflammatory microenvironment that favored Th1 immune response. However, this dramatic effect of EVs was transient and gradually faded ${ }^{130}$. Hence, optimum treatment time must be taken into consideration. Cheng et al. employed a peptide vaccine in combination with M1-like EVs in a melanoma model. These EVs were given $4 \mathrm{~h}$ before the peptide vaccine arrived at the tumor, and this combination therapy exhibited stronger antitumor effects compared to traditional adjuvants such as TLR agonist ${ }^{130}$. Mechanically, M1-like EVs might serve as an agent to stimulate proinflammatory milieu, which makes the immune system more sensitive to cancer vaccines, thereby inducing a superior antitumor effect. These findings strongly indicate that EVs from M1-like macrophages are a promising immune adjuvant suitable for cancer vaccines.

\section{Conclusions and future perspectives}

Notably, macrophages are key mediators in the innate immune system, and are involved in the pathology of many diseases. EVs are capable of exerting several functions similar to their parent cells, and have received increasing attention due to their natural portability and extraordinary actions on target cells. Similar to their parent cells, M $\phi$-EVs function extensively in the pathology of various diseases, as well as being robust mediators for immunotherapy, serving as therapeutics for many diseases. Employment of M $\phi$-EVs has several advantages. First, $M \phi-E V s$ can easily escape immunological surveillance due to abundant immune molecules such as CD47 on their surface, which help them escape immune attack $^{129}$. Second, the ability of M $\phi$-EVs to either enhance or suppress immune activity makes them attractive candidates for various diseases as discussed in this review. Third, administration of EVs as a substitute for $M \phi$ might 
reduce some of the risks associated with whole cell therapy such as cytokine release syndrome, also known as a cytokine storm ${ }^{136}$. Moreover, EVs are nanoscale and circulate readily compared to larger $M \phi$, and they can be further modified and used as potential drug delivery systems due to their ability to cross biological barriers ${ }^{137}$.

However, several limitations or issues raised by $\mathrm{M} \phi$-EVs should be taken into consideration before future clinical translation. After recognition of the pathogenicity of $\mathrm{M} \phi-$ EVs in diseases, it is crucial to further investigate how to regulate their release or their embedded contents properly to prevent development and progression of disease. Additionally, macrophages are able to exert immediate and diverse responses to different microenvironments, and this unique property seems important to their robust therapeutic effects in multiple diseases. However, unlike whole cells, M $\phi$-EVs alone are not believed to have similar action, and thus precise modulation of $\mathrm{M} \phi$-EV properties should be conducted in response to different diseases or conditions before intervention. Reprogramming or reengineering of $\mathrm{M} \phi$-EVs for disease therapy seems promising for the future, while the poor yield and/or limited function of M $\phi$-EVs using traditional methods has largely impaired their further biomedical applications, and thus new isolation or manufacture methods with higher yield/function of EVs are urgently needed. Nevertheless, these problems are not exclusively confined to macrophages and answers may come from academic studies devoted to the biological properties of EVs. Overall, the potential of macrophage-derived EVs in immunoregulation and disease intervention is highly promising.

\section{Acknowledgements}

This work was partly supported by grants from National Natural Science Foundation of China (81571808, 31871001, and 32071453), Sichuan Science and Technology Program (2019YJ0069), National Clinical Research Center for Geriatrics (Z20192002), and 1.3.5 Project for Disciplines of Excellence (ZYGD18014), West China Hospital. The authors would like to thank Haihong Xiao, Anqi Xu, and Xingwu Guan for technical assistance of figures preparation.

\section{Author details}

${ }^{1}$ Key Laboratory of Transplant Engineering and Immunology, Regenerative Medicine Research Center, National Clinical Research Center for Geriatrics, West China Hospital, Sichuan University, Chengdu, China. ${ }^{2}$ Institutes for Systems Genetics, Frontiers Science Center for Disease-related Molecular Network, West China Hospital, Sichuan University, Chengdu, China. ${ }^{3}$ Department of Critical Care Medicine, West China Hospital, Sichuan University, Chengdu, China

\section{Conflict of interest}

The authors declare that they have no conflict of interest.

\section{Publisher's note}

Springer Nature remains neutral with regard to jurisdictional claims in published maps and institutional affiliations.

Received: 3 August 2020 Revised: 8 October 2020 Accepted: 9 October 2020

Published online: 28 October 2020
References

1. Gentek, R., Molawi, K. \& Sieweke, M. H. Tissue macrophage identity and selfrenewal. Immunol. Rev. 262, 56-73 (2014).

2. Cao, Q., Harris, D. C. \& Wang, Y. Macrophages in kidney injury, inflammation, and fibrosis. Physiology 30, 183-194 (2015).

3. Mackaness, G. B. Cellular resistance to infection. J. Exp. Med. 116, 381-406 (1962).

4. Mackaness, G. B. The immunological basis of acquired cellular resistance. J. Exp. Med. 120, 105-120 (1964).

5. Liu, J., Wan, M., Lyon, C. J. \& Hu, T. Y. Nanomedicine therapies modulating Macrophage Dysfunction: a potential strategy to attenuate Cytokine Storms in severe infections. Theranostics 10, 9591-9600 (2020).

6. Reiner, N. E. Methods in molecular biology. Macrophages and dendritic cells. Methods and protocols. Preface. Methods Mol. Biol. 531, v-vi (2009).

7. Arango Duque, G. \& Descoteaux, A. Macrophage cytokines: involvement in immunity and infectious diseases. Front. Immunol. 5, 491 (2014).

8. Ding, A. H., Nathan, C. F. \& Stuehr, D. J. Release of reactive nitrogen intermediates and reactive oxygen intermediates from mouse peritoneal macrophages. Comparison of activating cytokines and evidence for independent production. J. Immunol. 141, 2407-2412 (1988).

9. Leopold Wager, C. M. \& Wormley, F. L. Jr Classical versus alternative macrophage activation: the Ying and the Yang in host defense against pulmonary fungal infections. Mucosal Immunol. 7, 1023-1035 (2014).

10. Nathan, C. \& Ding, A. Nonresolving inflammation. Cell 140, 871-882 (2010).

11. Chistiakov, D. A. et al. Macrophage phenotypic plasticity in atherosclerosis: the associated features and the peculiarities of the expression of inflammatory genes. Int. J. Cardiol. 184, 436-445 (2015).

12. Gundra, U. M. et al. Alternatively activated macrophages derived from monocytes and tissue macrophages are phenotypically and functionally distinct. Blood 123, e110-e122 (2014).

13. Zhang, W., Xu, W. \& Xiong, S. Blockade of Notch1 signaling alleviates murine lupus via blunting macrophage activation and M2b polarization. J. Immunol. 184, 6465-6478 (2010)

14. Lu, J. et al. Discrete functions of M2a and M2c macrophage subsets determine their relative efficacy in treating chronic kidney disease. Kidney Int. 84, 745-755 (2013).

15. Wynn, T. A., Chawla, A. \& Pollard, J. W. Macrophage biology in development, homeostasis and disease. Nature 496, 445-455 (2013).

16. Noy, R. \& Pollard, J. W. Tumor-associated macrophages: from mechanisms to therapy. Immunity 41, 49-61 (2014).

17. Cabarcas, S. M., Mathews, L. A. \& Farrar, W. L. The cancer stem cell niche-there goes the neighborhood? Int. J. Cancer 129, 2315-2327 (2011).

18. Zeisberger, S. M. et al. Clodronate-liposome-mediated depletion of tumourassociated macrophages: a new and highly effective antiangiogenic therapy approach. Br. J. Cancer Suppl. 95, 272-281 (2006).

19. Komohara, Y., Fujiwara, Y., Ohnishi, K. \& Takeya, M. Tumor-associated macrophages: potential therapeutic targets for anti-cancer therapy. Adv. Drug Deliv. Rev. 99, 180-185 (2016).

20. Chen, Y. et al. Tumor-associated macrophages: an accomplice in solid tumor progression. J. Biomed. Sci. 26, 78 (2019).

21. Miyachi, Y. et al. A reduced M1-like/M2-like ratio of macrophages in healthy adipose tissue expansion during SGLT2 inhibition. Sci. Rep. 8, 16113 (2018).

22. Jeppesen, D. K. et al. Reassessment of exosome composition. Cell 177, 428-445 (2019).

23. van Engeland, M., Nieland, L. J., Ramaekers, F. C., Schutte, B. \& Reutelingsperger, C. P. Annexin V-affinity assay: a review on an apoptosis detection system based on phosphatidylserine exposure. Cytometry 31, 1-9 (1998).

24. Xu, X., Lai, Y. \& Hua, Z. C. Apoptosis and apoptotic body: disease message and therapeutic target potentials. Biosci. Rep. 39, BSR20180992 (2019).

25. Zhang, $H$. et al. Identification of distinct nanoparticles and subsets of extracellular vesicles by asymmetric flow field-flow fractionation. Nat. Cell Biol. 20, 332-343 (2018).

26. Zhang, Q. et al. Transfer of functional cargo in exomeres. Cell Rep. 27, 940-954 (2019).

27. Di Vizio, D. et al. Oncosome formation in prostate cancer: association with a region of frequent chromosomal deletion in metastatic disease. Cancer Res. 69, 5601-5609 (2009).

28. Morello, M. et al. Large oncosomes mediate intercellular transfer of functional microRNA. Cell Cycle 12, 3526-3536 (2013). 
29. Josson, S. et al. Stromal fibroblast-derived miR-409 promotes epithelial-tomesenchymal transition and prostate tumorigenesis. Oncogene $\mathbf{3 4}$ 2690-2699 (2015).

30. Théry, C. et al. Minimal information for studies of extracellular vesicles 2018 (MISEV2018): a position statement of the International Society for Extracellular Vesicles and update of the MISEV2014 guidelines. J. Extracell. Vesicles 7, 1535750 (2018).

31. García-Manrique, P., Matos, M., Gutiérrez, G., Pazos, C. \& Blanco-López, M. C Therapeutic biomaterials based on extracellular vesicles: classification of bioengineering and mimetic preparation routes. J. Extracell. Vesicles 7, 1422676 (2018).

32. Wolf, $\mathrm{P}$. The nature and significance of platelet products in human plasma. Br. J. Haematol. 13, 269-288 (1967).

33. Mathieu, M., Martin-Jaular, L., Lavieu, G. \& Théry, C. Specificities of secretion and uptake of exosomes and other extracellular vesicles for cell-to-cell communication. Nat. Cell Biol. 21, 9-17 (2019).

34. Pegtel, D. M. \& Gould, S. J. Exosomes. Annu. Rev. Biochem. 88, 487-514 (2019).

35. Karpman, D., Ståhl, A. L. \& Arvidsson, I. Extracellular vesicles in renal disease. Nat. Rev. Nephrol. 13, 545-562 (2017).

36. Russell, A. E. et al. Biological membranes in EV biogenesis, stability, uptake, and cargo transfer: an ISEV position paper arising from the ISEV membranes and EVs workshop. J. Extracell. Vesicles 8, 1684862 (2019).

37. Kalluri, R. The biology and function of exosomes in cancer. J. Clin. Invest. 126 1208-1215 (2016).

38. Hessvik, N. P. \& Llorente, A. Current knowledge on exosome biogenesis and release. Cell Mol. Life Sci. 75, 193-208 (2018).

39. Stuffers, S., Sem Wegner, C., Stenmark, H. \& Brech, A. Multivesicular endosome biogenesis in the absence of ESCRTs. Traffic 10, 925-937 (2009).

40. Raposo, G. \& Stoorvogel, W. Extracellular vesicles: exosomes, microvesicles, and friends. J. Cell Biol. 200, 373-383 (2013).

41. Trajkovic, K. et al. Ceramide triggers budding of exosome vesicles into multivesicular endosomes. Science 319, 1244-1247 (2008).

42. Hemler, M. E. Tetraspanin functions and associated microdomains. Nat. Rev. Mol. Cell Biol. 6, 801-811 (2005)

43. Johnstone, R. M. Exosomes biological significance: a concise review. Blood Cells Mol. Dis. 36, 315-321 (2006).

44. Lebreton, A. \& Seraphin, B. Exosome-mediated quality control: substrate recruitment and molecular activity. Biochim. Biophys. Acta 1779, 558-565 (2008)

45. Mulcahy, L. A., Pink, R. C. \& Carter, D. R. Routes and mechanisms of extracellular vesicle uptake. J. Extracell. Vesicles 3, 24641 (2014).

46. Kowal, J., Tkach, M. \& Théry, C. Biogenesis and secretion of exosomes. Curr. Opin. Cell Biol. 29, 116-125 (2014).

47. Ostrowski, M. et al. Rab27a and Rab27b control different steps of the exosome secretion pathway. Nat. Cell Biol. 12, 19-30 (2010).

48. Wang, T. et al. Hypoxia-inducible factors and RAB22A mediate formation of microvesicles that stimulate breast cancer invasion and metastasis. Proc. Natl Acad. Sci. USA 111, E3234-E3242 (2014).

49. D'Souza-Schorey, C. \& Chavrier, P. ARF proteins: roles in membrane traffic and beyond. Nat. Rev. Mol. Cell Biol. 7, 347-358 (2006).

50. Singh, M. K. et al. A single class of ARF GTPase activated by several pathwayspecific ARF-GEFs regulates essential membrane traffic in Arabidopsis. PLoS Genet. 14, e1007795 (2018).

51. Muralidharan-Chari, V. et al. ARF6-regulated shedding of tumor cell-derived plasma membrane microvesicles. Curr. Biol. 19, 1875-1885 (2009).

52. Cocucci, E., Racchetti, G. \& Meldolesi, J. Shedding microvesicles: artefacts no more. Trends Cell Biol. 19, 43-51 (2009).

53. Escrevente, C., Keller, S., Altevogt, P. \& Costa, J. Interaction and uptake of exosomes by ovarian cancer cells. BMC Cancer 11 108-108 (2011).

54. Wu, K., Xing, F., Wu, S. Y. \& Watabe, K. Extracellular vesicles as emerging targets in cancer: Recent development from bench to bedside. Biochim. Biophys. Acta Rev. Cancer 1868, 538-563 (2017).

55. Clancy, J. W. et al. Regulated delivery of molecular cargo to invasive tumourderived microvesicles. Nat. Commun. 6, 6919 (2015).

56. Parolini, I. et al. Microenvironmental $\mathrm{pH}$ is a key factor for exosome traffic in tumor cells. J. Biol. Chem. 284, 34211-34222 (2009).

57. Gardiner, $C$. et al. Techniques used for the isolation and characterization of extracellular vesicles: results of a worldwide survey. J. Extracell. Vesicles $\mathbf{5}$ 32945 (2016).
58. Wu, K., Xing, F., Wu, S.-Y. \& Watabe, K. Extracellular vesicles as emerging targets in cancer: Recent development from bench to bedside. Biochim. Biophys. Acta Rev. Cancer 1868, 538-563 (2017).

59. Willms, E., Cabañas, C., Mäger, I., Wood, M. J. A. \& Vader, P. Extracellular vesicle heterogeneity: subpopulations, isolation techniques, and diverse functions in cancer progression. Front. Immunol. 9, 738 (2018).

60. Xu, R., Greening, D. W., Zhu, H.-J., Takahashi, N. \& Simpson, R. J. Extracellular vesicle isolation and characterization: toward clinical application. J. Clin. Invest. 126, 1152-1162 (2016)

61. Yang, F., Liao, X., Tian, Y. \& Li, G. Exosome separation using microfluidic systems: size-based, immunoaffinity-based and dynamic methodologies. Biotechnol. J. 12, 1600699 (2017).

62. Konoshenko, M. Y., Lekchnov, E. A., Vlassov, A. V. \& Laktionov, P. P. Isolation of extracellular vesicles: general methodologies and latest trends. Biomed. Res. Int. 2018, 8545347 (2018).

63. Carnino, J. M., Lee, H. \& Jin, Y. Isolation and characterization of extracellular vesicles from Broncho-alveolar lavage fluid: a review and comparison of different methods. Respir. Res. 20, 240 (2019).

64. Wu, B. et al. Separation and characterization of extracellular vesicles from human plasma by asymmetrical flow field-flow fractionation. Anal. Chim. Acta 1127, 234-245 (2020).

65. Sokolova, V. et al. Characterisation of exosomes derived from human cells by nanoparticle tracking analysis and scanning electron microscopy. Colloids Surf. B Biointerfaces 87, 146-150 (2011).

66. Feinberg, M. W. \& Moore, K. J. MicroRNA regulation of atherosclerosis. Circ. Res. 118, 703-720 (2016).

67. Koelwyn, G. J., Corr, E. M., Erbay, E. \& Moore, K. J. Regulation of macrophage immunometabolism in atherosclerosis. Nat. Immunol. 19, 526-537 (2018).

68. Tabas, I. \& Bornfeldt, K. E. Macrophage phenotype and function in different stages of atherosclerosis. Circ. Res. 118, 653-667 (2016).

69. Nguyen, M. A. et al. Extracellular vesicles secreted by atherogenic macrophages transfer microRNA to inhibit cell migration. Arterioscler. Thromb. Vasc Biol. 38, 49-63 (2018).

70. Huang, C. et al. Exosomes derived from oxidized LDL-stimulated macrophages attenuate the growth and tube formation of endothelial cells. Mol. Med. Rep. 17, 4605-4610 (2018).

71. Chen, L. et al. Exosomal IncRNA GAS5 regulates the apoptosis of macrophages and vascular endothelial cells in atherosclerosis. PLOS ONE 12, e0185406 (2017)

72. Koga, J. \& Aikawa, M. Crosstalk between macrophages and smooth muscle cells in atherosclerotic vascular diseases. Vasc. Pharmacol. 57, 24-28 (2012).

73. Niu, C. et al. Macrophage foam cell-derived extracellular vesicles promote vascular smooth muscle cell migration and adhesion. J. Am. Heart Assoc. 5, e004099 (2016).

74. Zhu, J. et al. Exosomes from nicotine-stimulated macrophages accelerate atherosclerosis through miR-21-3p/PTEN-mediated VSMC migration and proliferation. Theranostics 9, 6901-6919 (2019).

75. Haudek, S. B. et al. Monocytic fibroblast precursors mediate fibrosis in angiotensin-II-induced cardiac hypertrophy. J. Mol. Cell Cardiol. 49, 499-507 (2010).

76. Ren, J. et al. Proinflammatory protein CARD9 is essential for infiltration of monocytic fibroblast precursors and cardiac fibrosis caused by Angiotensin II infusion. Am. J. Hypertens. 24, 701-707 (2011).

77. Wang, C. et al. Macrophage-derived mir-155-containing exosomes suppress fibroblast proliferation and promote fibroblast inflammation during cardiac injury. Mol. Ther. 25, 192-204 (2017).

78. Osada-Oka, M. et al. Macrophage-derived exosomes induce inflammatory factors in endothelial cells under hypertensive conditions. Hypertens. Res. $\mathbf{4 0}$ 353-360 (2017).

79. Feng, Z. et al. Tumor-associated macrophage-derived exosomal microRNA$155-5 p$ stimulates intracranial aneurysm formation and macrophage infiltration. Clin. Sci. 133, 2265-2282 (2019).

80. Kassi, E., Pervanidou, P., Kaltsas, G. \& Chrousos, G. Metabolic syndrome: definitions and controversies. BMC Med 9, 48 (2011).

81. Smith, U. Abdominal obesity: a marker of ectopic fat accumulation. J. Clin. Invest. 125, 1790-1792 (2015).

82. Khodabandehloo, H., Gorgani-Firuzjaee, S., Panahi, G. \& Meshkani, R. Molecular and cellular mechanisms linking inflammation to insulin resistance and beta-cell dysfunction. Transl. Res. 167, 228-256 (2016).

83. Russo, L. \& Lumeng, C. N. Properties and functions of adipose tissue macrophages in obesity. Immunology 155, 407-417 (2018). 
84. Tsuruta, M. et al. Metabolic endotoxemia-activated macrophages promote pancreatic $\beta$ cell death via IFNß-Xaf1 pathway. Horm. Metab. Res. 50, 160-167 (2018).

85. Liu, T., Sun, Y. C., Cheng, P. \& Shao, H. G. Adipose tissue macrophage-derived exosomal miR-29a regulates obesity-associated insulin resistance. Biochem. Biophys. Res. Commun. 515, 352-358 (2019).

86. Ying, W. et al. Adipose tissue macrophage-derived exosomal miRNAs can modulate in vivo and in vitro insulin sensitivity. Cell 171, 372-384 (2017).

87. De Silva, N., Samblas, M., Martinez, J. A. \& Milagro, F. I. Effects of exosomes from LPS-activated macrophages on adipocyte gene expression, differentiation, and insulin-dependent glucose uptake. J. Physiol. Biochem. 74 559-568 (2018).

88. Goulielmaki, E. et al. Tissue-infiltrating macrophages mediate an exosomebased metabolic reprogramming upon DNA damage. Nat. Commun. 11, 42 (2020).

89. Floyd, K., Glaziou, P., Zumla, A. \& Raviglione, M. The global tuberculosis epidemic and progress in care, prevention, and research: an overview in year 3 of the End TB era. Lancet Respir. Med. 6, 299-314 (2018).

90. Bold, T. D. \& Ernst, J. D. Who benefits from granulomas, mycobacteria or host? Cell 136, 17-19 (2009).

91. Singh, P. P., Smith, V. L., Karakousis, P. C. \& Schorey, J. S. Exosomes isolated from mycobacteria-infected mice or cultured macrophages can recruit and activate immune cells in vitro and in vivo. J. Immunol. 189, 777-785 (2012).

92. Roth, W. W., Huang, M. B., Addae Konadu, K., Powell, M. D. \& Bond, V. C. Micro RNA in exosomes from HIV-infected macrophages. Int. J. Environ. Res. Public Health 13, 32 (2015).

93. Kadiu, I., Narayanasamy, P., Dash, P. K., Zhang, W. \& Gendelman, H. E. Biochemical and biologic characterization of exosomes and microvesicles as facilitators of HIV-1 infection in macrophages. J. Immunol. 189, 744-754 (2012).

94. Triplette, M., Crothers, K. \& Attia, E. F. Non-infectious pulmonary diseases and HIV. Curr. HIV/AIDS Rep. 13, 140-148 (2016).

95. Kamil, F. \& Rowe, J. H. How does the tumor microenvironment play a role in hepatobiliary tumors? J. Gastrointest. Oncol. 9, 180-195 (2018).

96. Zhou, J. et al. Exosomes released from tumor-associated macrophages transfer miRNAs that induce a Treg/Th17 cell imbalance in epithelial ovarian cancer. Cancer Immunol. Res. 6, 1578-1592 (2018).

97. Yin, Z. et al. Macrophage-derived exosomal microRNA-501-3p promotes progression of pancreatic ductal adenocarcinoma through the TGFBR3mediated TGF- $\beta$ signaling pathway. J. Exp. Clin. Cancer Res. 38, 310 (2019).

98. Zhu, X. et al. Macrophages derived exosomes deliver miR-223 to epithelial ovarian cancer cells to elicit a chemoresistant phenotype. J. Exp. Clin. Cancer Res. 38, 81 (2019).

99. Cianciaruso, C. et al. Molecular profiling and functional analysis of macrophage-derived tumor extracellular vesicles. Cell Rep. 27, 3062-3080 (2019)

100. Wynn, T. A. \& Vannella, K. M. Macrophages in tissue repair, regeneration, and fibrosis. Immunity 44, 450-462 (2016).

101. Smigiel, K. S. \& Parks, W. C. Macrophages, wound healing, and fibrosis: recent insights. Curr. Rheumatol. Rep. 20, 17 (2018).

102. Cui, $\mathrm{H}$. et al. Macrophage-derived miRNA-containing exosomes induce peritendinous fibrosis after tendon injury through the miR-21-5p/Smad7 pathway. Mol. Ther. Nucleic Acids 14, 114-130 (2019).

103. Munson, P., Lam, Y. W., Dragon, J., MacPherson, M. \& Shukla, A. Exosomes from asbestos-exposed cells modulate gene expression in mesothelial cells. FASEB J. 32, 4328-4342 (2018).

104. Wang, D. et al. Exosomal miR-125a-5p derived from silica-exposed macrophages induces fibroblast transdifferentiation. Ecotoxicol. Environ. Saf. 192, 110253 (2020)

105. Yao, M. Y. et al. microRNA-328 in exosomes derived from M2 macrophages exerts a promotive effect on the progression of pulmonary fibrosis via FAM13A in a rat model. Exp. Mol. Med. 51, 1-16 (2019).

106. Chen, J. et al. Blockade of IncRNA-ASLNCS5088-enriched exosome generation in M2 macrophages by GW4869 dampens the effect of M2 macrophages on orchestrating fibroblast activation. FASEB J. 33, 12200-12212 (2019).

107. Zhu, Q. J., Zhu, M., Xu, X. X., Meng, X. M. \& Wu, Y. G. Exosomes from high glucose-treated macrophages activate glomerular mesangial cells via TGF- $\beta 1 / \mathrm{Smad} 3$ pathway in vivo and in vitro. FASEB J. 33, 9279-9290 (2019).
108. Govindappa, P. K. et al. Targeting exosome-associated human antigen R attenuates fibrosis and inflammation in diabetic heart. FASEB J. 34, 2238-2251 (2020).

109. Samanta, S. et al. Exosomes: new molecular targets of diseases. Acta Pharmacol. Sin. 39, 501-513 (2018).

110. Matsumoto, J., Stewart, T., Banks, W. A. \& Zhang, J. The transport mechanism of extracellular vesicles at the blood-brain barrier. Curr. Pharmaceut. Des. 23, 6206-6214 (2017).

111. Dai, Y. et al. M2 macrophage-derived exosomes carry microRNA-148a to alleviate myocardial ischemia/reperfusion injury via inhibiting TXNIP and the TLR4/NF-KB/NLRP3 inflammasome signaling pathway. J. Mol. Cell Cardiol. 142, 65-79 (2020).

112. Li, M. et al. Macrophage-derived exosomes accelerate wound healing through their anti-inflammation effects in a diabetic rat model. Artif. Cells Nanomed. Biotechnol. 47, 3793-3803 (2019).

113. Rajendran, R. L. et al. Macrophage-derived extracellular vesicle promotes hair growth. Cells 9, 856 (2020).

114. Saha, S. et al. Macrophage-derived extracellular vesicle-packaged WNTs rescue intestinal stem cells and enhance survival after radiation injury. Nat. Commun. 7, 13096 (2016).

115. Yang, R. et al. Exosomes derived from M2b macrophages attenuate DSSinduced colitis. Front. Immunol. 10, 2346 (2019).

116. Kim, H. et al. Exosome-guided phenotypic switch of M1 to M2 macrophages for cutaneous wound healing. Adv. Sci. 6, 1900513 (2019).

117. Bhatnagar, S., Shinagawa, K., Castellino, F. J. \& Schorey, J. S. Exosomes released from macrophages infected with intracellular pathogens stimulate a proinflammatory response in vitro and in vivo. Blood 110, 3234-3244 (2007).

118. Cheng, Y. \& Schorey, J. S. Extracellular vesicles deliver Mycobacterium RNA to promote host immunity and bacterial killing. EMBO Rep. 20, e46613 (2019).

119. Cheng, Y. \& Schorey, J. S. Exosomes carrying mycobacterial antigens can protect mice against Mycobacterium tuberculosis infection. Eur. J. Immunol. 43, 3279-3290 (2013).

120. Giri, P. K., Kruh, N. A., Dobos, K. M. \& Schorey, J. S. Proteomic analysis identifies highly antigenic proteins in exosomes from M. tuberculosis-infected and culture filtrate protein-treated macrophages. Proteomics 10, 3190-3202 (2010).

121. Reales-Calderón, J. A., Vaz, C., Monteoliva, L., Molero, G. \& Gil, C. Candida albicans modifies the protein composition and size distribution of THP-1 macrophage-derived extracellular vesicles. J. Proteome Res. 16, 87-105 (2017).

122. Velandia-Romero, M. L. et al. Extracellular vesicles of U937 macrophage cell line infected with DENV-2 induce activation in endothelial cells EA.hy926. PLOS ONE 15, e0227030 (2020).

123. Zhou, Y. et al. Toll-like receptor 3-activated macrophages confer anti-HCV activity to hepatocytes through exosomes. FASEB J. 30, 4132-4140 (2016).

124. Cai, C. et al. Macrophage-derived extracellular vesicles induce long-lasting immunity against hepatitis $C$ virus which is blunted by polyunsaturated fatty acids. Front. Immunol. 9, 723 (2018).

125. Record, M., Carayon, K., Poirot, M. \& Silvente-Poirot, S. Exosomes as new vesicular lipid transporters involved in cell-cell communication and various pathophysiologies. Biochim. Biophys. Acta 1841, 108-120 (2014).

126. El Andaloussi, S., Lakhal, S., Mäger, I. \& Wood, M. J. A. Exosomes for targeted siRNA delivery across biological barriers. Adv. Drug Deliv. Rev. 65, 391-397 (2013).

127. Bolukbasi, M. F. et al. miR-1289 and "Zipcode"-like Sequence Enrich mRNAs in Microvesicles. Mol. Ther. Nucleic Acids 1, e10 (2012).

128. Kaur, S. et al. CD47-dependent immunomodulatory and angiogenic activities of extracellular vesicles produced by T cells. Matrix Biol. 37, 49-59 (2014).

129. Kamerkar, S. et al. Exosomes facilitate therapeutic targeting of oncogenic KRAS in pancreatic cancer. Nature 546, 498-503 (2017).

130. Cheng, L., Wang, Y. \& Huang, L. Exosomes from M1-polarized macrophages potentiate the cancer vaccine by creating a pro-inflammatory microenvironment in the lymph node. Mol. Ther. 25, 1665-1675 (2017).

131. Kim, M. S. et al. Engineering macrophage-derived exosomes for targeted paclitaxel delivery to pulmonary metastases: in vitro and in vivo evaluations. Nanomedicine 14, 195-204 (2018).

132. Haney, M. J. et al. Macrophage-derived extracellular vesicles as drug delivery systems for Triple Negative Breast Cancer (TNBC) therapy. J. Neuroimmune Pharmacol. 15, 487-500 (2019).

133. Li, S. et al. Engineering macrophage-derived exosomes for targeted chemotherapy of triple-negative breast cancer. Nanoscale 12, 10854-10862 (2020). 
134. Rayamajhi, S., Nguyen, T. D. T., Marasini, R. \& Aryal, S. Macrophagederived exosome-mimetic hybrid vesicles for tumor targeted drug delivery. Acta Biomater. 94, 482-494 (2019).

135. Finn, O. J. Cancer immunology. New Engl. J. Med. 358, 2704-2715 (2008).
136. Schulert, G. S. \& Grom, A. A. Pathogenesis of macrophage activation syndrome and potential for cytokine- directed therapies. Annu. Rev. Med. 66 145-159 (2015).

137. Formicola, B., Cox, A., Dal Magro, R., Masserini, M. \& Re, F. Nanomedicine for the treatment of Alzheimer's disease. J. Biomed. Nanotechnol. 15, 1997-2024 (2019) 